University of Wollongong

Research Online

Australian Institute for Innovative Materials -

Papers

Australian Institute for Innovative Materials

$1-1-2019$

\title{
Sb2Te3 topological insulator: Surface plasmon resonance and application in refractive index monitoring
}

Hua Lu

Northwestern Polytechnical University

Siqing Dai

Northwestern Polytechnical University

Zengji Yue

University of Wollongong, zengji@uow.edu.au

Yicun Fan

Northwestern Polytechnical University

Huachao Cheng

Northwestern Polytechnical University

See next page for additional authors

Follow this and additional works at: https://ro.uow.edu.au/aiimpapers

Part of the Engineering Commons, and the Physical Sciences and Mathematics Commons

Research Online is the open access institutional repository for the University of Wollongong. For further information contact the UOW Library: research-pubs@uow.edu.au 


\title{
Sb2Te3 topological insulator: Surface plasmon resonance and application in refractive index monitoring
}

\author{
Abstract \\ Topological insulators as new emerging building blocks in electronics and photonics present promising \\ prospects for exciting surface plasmons and enhancing light-matter interaction. Thus, exploring the \\ visible-range plasmonic response of topological insulators is significant to reveal their optical \\ characteristics and broaden their applications at high frequencies. Herein, we report the experimental \\ demonstration of a visible-range surface plasmon resonance (SPR) effect on an antimony telluride (Sb 2 \\ Te 3 ) topological insulator film. The results show that the SPR can be excited with a relatively small \\ incident angle in the Kretschmann configuration based on the Sb 2 Te 3 film. Especially, we develop an \\ impactful digital holographic imaging system based on the topological insulator SPR and realize the \\ dynamic monitoring of refractive index variation. Compared with the traditional SPR, the Sb 2 Te 3 -based \\ SPR possesses a broader measurement range. Our findings open a new avenue for exploring the optical \\ physics and practical applications of topological insulators, such as environmental and biochemical \\ sensing. \\ Disciplines \\ Engineering | Physical Sciences and Mathematics

\section{Publication Details} \\ Lu, H., Dai, S., Yue, Z., Fan, Y., Cheng, H., Di, J., Mao, D., Li, E., Mei, T. \& Zhao, J. (2019). Sb2Te3 topological \\ insulator: Surface plasmon resonance and application in refractive index monitoring. Nanoscale, 11 (11), \\ 4759-4766.
}

\section{Authors}

Hua Lu, Siqing Dai, Zengji Yue, Yicun Fan, Huachao Cheng, Jianglei Di, Dong Mao, Enpu Li, Ting Mei, and Jianlin Zhao 


\title{
$\mathrm{Sb}_{2} \mathrm{Te}_{3}$ Topological Insulator: Surface Plasmon Resonance and Application in Refractive Index Monitoring
}

\author{
Hua Lu, ${ }^{1, *}$ Siqing Dai, ${ }^{1}$ Zengji Yue, ${ }^{2}$ Yicun Fan, ${ }^{1}$ Huachao Cheng, ${ }^{1}$ Jianglei Di, ${ }^{1}$ Dong Mao, ${ }^{1}$ \\ Enpu $\mathrm{Li},{ }^{1}$ and Jianlin $\mathrm{Zhao}^{1, *}$
}

${ }^{1}$ MOE Key Laboratory of Material Physics and Chemistry under Extraordinary Conditions, and Shaanxi Key Laboratory of Optical Information Technology, School of Science,

Northwestern Polytechnical University, Xi'an 710072, China

${ }^{2}$ Institute for Superconducting \& Electronic Materials, University of Wollongong, North

Wollongong, New South Wales 2500, Australia

*E-mail: hualu@nwpu.edu.cn

*E-mail: jlzhao@nwpu.edu.cn

Topological insulators as new emerging building blocks in electronics and photonics have presented promising prospects for exciting surface plasmons and enhancing light-matter interaction. Exploring the visible range plasmonic response of topological insulator family members is of great importance for revealing the optical characteristics of topological insulators and broadening their significant applications at high frequencies. Here, we firstly, to the best of our knowledge, demonstrate the surface plasmon resonance (SPR) effect on the antimony telluride $\left(\mathrm{Sb}_{2} \mathrm{Te}_{3}\right)$ topological insulator film. The results show that the SPR can be excited with the relatively small incident angle in the Kretschmann configuration based on the $\mathrm{Sb}_{2} \mathrm{Te}_{3}$ film with the metal-like surface state at visible frequencies. Especially, we develop an impactful digital holographic imaging system based on the topological insulator SPR and realize the dynamic monitoring of refractive index variation. Compared with the traditional SPR, the $\mathrm{Sb}_{2} \mathrm{Te}_{3}$-based SPR possesses broader measurement range. Our findings will open up a new avenue for exploring optical physics and practical applications of topological insulators, such as environmental and biochemical sensing. 
Surface plasmons (SPs), light-driven oscillation of free electrons along the conductor-dielectric interface, play a crucial role in current development of optical physics and functional devices due to the remarkable capabilities of confining light at the subwavelength scale, enhancing the near-field intensity, and overcoming the diffraction limit of light. ${ }^{[1-14]}$ Over the past two decades, numerous unusual optical phenomena were observed in metal-based plasmonic systems, such as extraordinary optical transmission, ${ }^{[15]}$ plasmonic guiding, ${ }^{[3]}$ plasmonic resonance, ${ }^{[16]}$ Optically-triggered memory effect, ${ }^{[6]}$ meta-holography, ${ }^{[7]}$ Raman scattering ${ }^{[8]}$ optical activity, ${ }^{[9]}$ nonlinear enhancement, ${ }^{[10,17]}$ plasmonic trapping, ${ }^{[11]}$ phase manipulation, ${ }^{[18]}$ plasmon-induced transparency, ${ }^{[19,}{ }^{20]}$ quantum plasmonics, ${ }^{[21]}$ and plasmonic focusing. ${ }^{[22,23]}$ Based on these plasmonic responses, a large number of optical functionalities were proposed and investigated, for example nanolasers, ${ }^{[12,}{ }^{24]}$ holographic imaging, ${ }^{[13]}$ optical detection, ${ }^{[14]}$ perfect absorber, ${ }^{[25]}$ all-optical switches,${ }^{[26]}$ color display, ${ }^{[27]}$ optical modulation, ${ }^{[28]}$ enhanced Raman spectroscopy, ${ }^{[29]}$ and highly sensitive sensing. ${ }^{[30]}$ Recently, the new emerging nanomaterials provide exciting opportunities for the development of plasmonics and related technologies. ${ }^{[31]}$ After graphene was successfully exfoliated from graphite, this single-atomic-layer material significantly extended the operating range of plasmonics from the visible/near-infrared to the mid-infrared/terahertz. ${ }^{[32-37]}$ The strong light confinement and flexible tunability make graphene plasmonics a promising candidate for the realization of optical devices. ${ }^{[34,35]}$ The plasmonic activities were also observed in molybdenum disulfide ${ }^{[38]}$ and black phosphorus. ${ }^{[39,40]}$ Quite recently, the topological insulators as new building blocks in electrics and photonics have attracted particular attentions in the field of plasmonics due to the topologically protected metallic surface state 
caused by the strong spin-orbit coupling in a ultra-broad range from the ultraviolet to the terahertz. ${ }^{[1-50]}$ Until now, the topological insulator materials containing $\mathrm{Bi}_{2} \mathrm{Se}_{3},{ }^{[41,42]}$ $\mathrm{Bi}_{2} \mathrm{Te}_{3},{ }^{[43,44]}$ and $\mathrm{Bi}_{1.5} \mathrm{Sb}_{0.5} \mathrm{Te}_{1.8} \mathrm{Se}_{1.2}{ }^{[45,46]}$ have experimentally exhibited exciting plasmonic behaviors in an ultrabroad region from the ultraviolet to the terahertz. Lupi et al. demonstrated the plasmonic excitation and magnetoplasmons from the microribbon arrays of $\mathrm{Bi}_{2} \mathrm{Se}_{3}$ topological insulator at terahertz frequencies. ${ }^{[41,42]}$ By using SNOM, Bao et al. achieved the mid-infrared plasmonic response in the $\mathrm{Bi}_{2} \mathrm{Se}_{3}$ nanosheets. ${ }^{[44]}$ Zheludev et al., for the first time, reported the exciting plasmonic response at visible frequencies in the structures based on the topological insulator $\mathrm{Bi}_{1.5} \mathrm{Sb}_{0.5} \mathrm{Te}_{1.8} \mathrm{Se}_{1.2 .}{ }^{[45-47]}$ Especially, they observed near-field visible plasmon excitation in the $\mathrm{Bi}_{1.5} \mathrm{Sb}_{0.5} \mathrm{Te}_{1.8} \mathrm{Se}_{1.2}$ nanodisk and nanoflake by using scattering-type scanning near-field optical microscope (s-SNOM). ${ }^{[46]}$ Loh et al. observed the visible surface plasmon polariton modes in single $\mathrm{Bi}_{2} \mathrm{Te}_{3}$ nanoplate via transmission electron microscopy-based electron energyloss spectroscopy/cathodoluminescence spectroscopy. ${ }^{[43]}$ Subsequently, Yue et al. demonstrated the visible plasmonic resonance and backward light scattering was observed in the $\mathrm{Bi}_{1.5} \mathrm{Sb}_{0.5} \mathrm{Te}_{1.8} \mathrm{Se}_{1.2}$ nanocone arrays. ${ }^{[48]}$ The high-frequency plasmonic response in visible region makes topological insulators integratable with photonics and electronics, enabling the realization of novel optical and optoelectrical functionalities, such as enhanced photoluminescence and energy harvesting. ${ }^{[48,50]}$ Even so, plasmonic behaviors in another primary topological insulator $\mathrm{Sb}_{2} \mathrm{Te}_{3}$ have rarely been reported.

Here, we firstly report the visible-range plasmonic resonance in the nanofilm structure based on the $\mathrm{Sb}_{2} \mathrm{Te}_{3}$ topological insulator. Both the experimental and theoretical results demonstrate that the surface plasmon resonance (SPR) can be effectively excited on 
the $\mathrm{Sb}_{2} \mathrm{Te}_{3}$ film in the simple Kretschmann configuration with the relatively small angle at visible frequencies. Moreover, we innovationally combine a developed digital holographic imaging system with the topological insulator-based SPR and achieve the dynamic monitoring of near-field refractive index variation. Compared with the traditional metal-based $\mathrm{SPR}$, the $\mathrm{Sb}_{2} \mathrm{Te}_{3}$-based SPR presents broader measurement range. These results will open a new door for the investigation of fundamental science and applications of topological insulators in photonics, plasmonics, and optoelectronics.

The material $\mathrm{Sb}_{2} \mathrm{Te}_{3}$ employed in the experiments has been confirmed as a primary family member of topological insulators. ${ }^{[51]}$ As shown in Figure 1a, the $\mathrm{Sb}_{2} \mathrm{Te}_{3}$ thin film is grown on the uniform glass substrate employing the atomic layer deposition (ALD) method (see details in the Experimental Section). From Figure 2a, we can see that the $\mathrm{Sb}_{2} \mathrm{Te}_{3}$ film deposited by ALD on the glass maintains good continuity over a relatively large area and possesses good flatness on the micrometer scale. The $\mathrm{Sb}_{2} \mathrm{Te}_{3}$ film is deposited as $50 \mathrm{~nm}$ thickness, which can be verified by the characterization results, as shown in Figure 2b. As depicted in Figure 2c, the Raman shift spectrum of $\mathrm{Sb}_{2} \mathrm{Te}_{3}$ film possesses the obvious $\mathrm{E}_{\mathrm{g}}^{2}$ peak at $122 \mathrm{~cm}^{-1}$ and $\mathrm{A}_{1 \mathrm{~g}}^{2}$ peak at $141 \mathrm{~cm}^{-1}$, which agrees well with the previous results. ${ }^{[52,53]}$ The Raman spectrum confirms the $\mathrm{Sb}_{2} \mathrm{Te}_{3}$ stoichiometry of the film. ${ }^{[52]}$ The chemical composition of the film is confirmed as the $\mathrm{Sb}$ and Te elements by the energy-dispersive X-ray spectroscopy (EDS) measurement, as shown in Figure 2d. The grown $\mathrm{Sb}_{2} \mathrm{Te}_{3}$ material possesses good crystalline character, which can be confirmed by X-ray diffraction (XRD) measurement (Supporting Information). The optical parameters of the $\mathrm{Sb}_{2} \mathrm{Te}_{3}$ topological insulator can be measured by the spectroscopic ellipsometer. As depicted in the inset of 
Figure 1b, the topological insulator hold metallic surface state and insulating bulk state, which demonstrate different optical properties. ${ }^{[45,52]}$ The dielectric constants of the surface and bulk layers $\left(\varepsilon_{s}\right.$ and $\left.\varepsilon_{b}\right)$ can be fitted by the Drude and Tauc-Lorentz models in the experimental measurement, respectively (Supporting information) ${ }^{[45]}$ Figure $\mathbf{1 b}$ depicts the wavelength-dependent dielectric dispersion of the surface and bulk layers achieved using the fitted parameters. The real part of dielectric constant for the surface layer represents negative value $\left(\varepsilon_{s}^{\prime}<0\right)$ in the visible region. This property similar to the noble metals (e.g. gold and silver) denotes that the surface layer of $\mathrm{Sb}_{2} \mathrm{Te}_{3}$ topological insulator is able to support the SPs at visible wavelengths. ${ }^{[1-3]}$ For the bulk layer, the real part of dielectric constant is negative $\left(\varepsilon_{b}{ }^{\prime}<0\right)$ when the wavelength is less than $553 \mathrm{~nm}$, while positive $\left(\varepsilon_{b}{ }^{\prime}>0\right)$ at the long wavelengths. Thus, the bulk layer of the $\mathrm{Sb}_{2} \mathrm{Te}_{3}$ can be regarded as an insulator at the long wavelength in the visible region. In Figure 1b, the total dielectric constant measured by the spectroscopic ellipsometer possesses the negative real part in the entire visible region, ${ }^{[54]}$ which illustrates that the $\mathrm{Sb}_{2} \mathrm{Te}_{3}$ will remain the metal-like feature at visible wavelengths. The imagery part of $\mathrm{Sb}_{2} \mathrm{Te}_{3}$ dielectric constant can be seen in Supporting Information.

The Kretschmann configuration provides a simple and valid method for excitation of SPs ${ }^{[55]}$ When the incident light satisfies the wavevector matching condition with SPs, It will strongly couple with SPs via the evanescent field and generate the SPR on the metal film. The reflection spectrum will form a distinct dip at the SPR excitation wavelength or angle. We establish an optical path to experimentally demonstrate the generation of the SPR supported by the $\mathrm{Sb}_{2} \mathrm{Te}_{3}$ thin film. As depicted in Figure 3a, the experimental setup contains the laser source, optical fiber coupler (OFC), expanding lens (EL), aperture stop (AS), collimator lens 
(CL), linear polarizer (LP), rectangular prism (RP), and photodiode-based detector. The OFC, EL, AS, and CL are used to collimate the monochromatic beam from the visible laser source. The AS and LP enable to control the size and polarization state of the incident light beam, respectively. The glass slide is adhered to the RP (K9 glass) using the refractive index matching liquid (Cargille Laboratories, $n=1.516$ ). The detector is employed to record the power of reflected light from the $\mathrm{Sb}_{2} \mathrm{Te}_{3}$ thin film. The light reflection at different incident angles can be measured by rotating the RP placed on a rotator. As depicted in the inset of Figure 3a, $\theta_{e}$ and $\theta_{i}$ stand for the external and internal incident angles, respectively.

Figure $3 \mathrm{~b}$ depicts the experiment results of the incident angle-dependent reflection from the Kretschmann configuration with the $\mathrm{Sb}_{2} \mathrm{Te}_{3}$ thin film. It shows that the $p$-polarized light beams with wavelengths of 632.8 and $640 \mathrm{~nm}$ present the distinct reflection dips at $\theta_{e} \approx 39.5^{\circ}$ and $38.8^{\circ}$, respectively. However, the reflection is insensitive to the incident angle for the $s$-polarized light (Supporting Information). Thus, the reflection dips for $p$-polarized light can be attributed to the excitation of the SPR on the surface of $\mathrm{Sb}_{2} \mathrm{Te}_{3}$ thin film at these wavelengths. To verify it, we theoretically calculate the SPR dispersion characteristic of the $\mathrm{Sb}_{2} \mathrm{Te}_{3}$ thin film in the visible region (see Experimental Section). As shown in Figure 3c, the angle of SPR excitation monotonically decreases with increasing the operating wavelength. The theoretical calculations are basically consistent with the experiment results. To further clarify the SPR excitation, we numerically simulate the electric field distribution in the $\mathrm{Sb}_{2} \mathrm{Te}_{3}$ film structure by the finite-difference time-domain method (see Experimental Section). As depicted in the inset of Figure 3c, the p-polarized incident light with a wavelength of $640 \mathrm{~nm}$ simulates a distinct SPR on the $\mathrm{Sb}_{2} \mathrm{Te}_{3}$ thin film at the excitation angle. It is worth noting that 
the $\mathrm{Sb}_{2} \mathrm{Te}_{3}$-based SPR exhibits the strong field penetration in air, which contributes to the effective sensing of surrounding environment. Moreover, we find that the SPR excitation angle of the $\mathrm{Sb}_{2} \mathrm{Te}_{3}$ thin film is relatively small, compared with that of traditional metal-based SPR (Supporting Information). The SPR response is capable of particular applications in refractive index sensing, monitoring and measurement due to the high surface sensitivity. ${ }^{[56-59]}$ However, the traditional SPR in Kretschmann configurations suffers from the large incident angle, resulting in the limited testing range for specimens. The $\mathrm{Sb}_{2} \mathrm{Te}_{3}$-based SPR could offer a feasible avenue to solve this problem and facilitate the detection of environmental refractive index with a smaller angle or broader measuring range (Supporting Information). This feature will promote the potential of topological insulator plasmonics in practical applications on biochemical and environmental sensing. For example, the SPR reflection intensity or phase will change with the alternation of environmental refractive index on the topological insulator film, contributing to the detection of DNA, chemical contaminants, gases, and so on. ${ }^{[60]}$ The topological insulators can find crucial applications in photothermal conversion. ${ }^{[61]}$ The plasmonic response in the topological insulators enables the enhancement of photothermal conversion. ${ }^{[62]}$ The surface properties of this topological insulator may provide another pathway for the relatively new optical physics, especially Fano resonance, ${ }^{[63]}$ plasmon-induced transparency, ${ }^{[64]}$ Klein tunneling, ${ }^{[65]}$ and twisted Ruderman-Kittel-KasuyaYosida (RKKY) interaction. ${ }^{[66]}$

The SPR-based sensing in the Kretschmann configuration is mainly derived from the alteration of reflection intensity with changing the refractive index of the specimen. Actually, the reflection phase change can also play a crucial role in the optical sensing ${ }^{[58]}$ Fortunately, 
DH can simultaneously provide the intensity and phase information of the specimens with the advantages of dynamic and non-intrusive measurement. ${ }^{[59,67]}$ The SPR-based DH enables to measure the near-field refractive index distribution of the specimen from the hologram recorded in the far field. ${ }^{[59]} \mathrm{In} \mathrm{DH}$, the intensity and phase information of the tested specimen are obtained with the angular spectrum reconstruction method (Supporting Information). Based on the principle of double-exposure holographic interferometry (Supporting Information), the phase differences of reconstructed object waves and intensity ratios at different time $t$ can be calculated (see Experimental Section). More specifically, the phase difference can be expressed as $\Delta \phi_{O t}=\phi_{O t^{-}} \phi_{O 0}=\arg \left(O_{t} / O_{0}\right)$, where $\phi_{O t}$ and $\phi_{O 0}$ stand for the phase distributions of object waves at time $t$ and the start time ( $t=0)$, respectively. $O_{t}$ and $O_{0}$ are the reconstructed object waves at time $t$ and reference object wave at the start time, respectively. The intensity ratio (reflection) can be calculated using the formula $I R_{t}=\left|O_{t} / O_{0}\right|^{2}$.

Here, we innovatively introduce the topological insulator-based SPR with DH to achieve the real-time monitoring of the refractive index variation. Figure 4 depicts a simple and compact experimental setup to realize the SPR holographic imaging for the dynamic detection of refractive index variation of an ethanol-water mixture. For the ethanol-water mixture, the ratio of pure ethanol and deionized water is set as 1:1. Thus, the refractive index of the mixture will change from 1.350 to 1.331 when the ethanol volatilizes at room temperature. As shown in Figure 3a, we employ the Wollaston prism (WP), LP, and CCD to replace the detector for building up a robust common-path digital holographic imaging system. The laser beam with $640 \mathrm{~nm}$ wavelength and $45^{\circ}$ polarization obliquely impinges in the Kretschmann configuration. When the specimen (mixture droplet) is injected on the $\mathrm{Sb}_{2} \mathrm{Te}_{3}$ film (Figure 4, 
upper-right), the SPR will be generated when the laser beam is incident at a specific angle. The reflected light containing the specimen information is projected on the CCD camera after passing through the WP and LP. The $s$ - and $p$-polarized components of the reflected light beam are split with a small angle by the WP for recording off-axis holograms (Figure 4, lower-right). The $p$-polarized component can couple with the SPR on the $\mathrm{Sb}_{2} \mathrm{Te}_{3}$ film, which is sensitive to the refractive index variation of injected droplet. However, the s-polarized component is unable to carry the information of the object (droplet) due to the forbidden SPR excitation. Thus, the $s$ - and $p$-polarized components can be respectively treated as the reference and object waves, as shown in Figure 1a. The two split light beams will possess the same polarization state after passing the LP and interfere with each other on the CCD target plane to form the holograms. We can control the beam size of incident light by adjusting the AS and achieve the full-view hologram of the specimen.

Generally, the incident laser beam should be fixed near the SPR excitation angle for high-sensitivity measurement of refractive index variation. To select a proper incident angle, we inject a deionized water droplet on the $\mathrm{Sb}_{2} \mathrm{Te}_{3}$ film and measure the angle-dependent reflection of light beam $(640 \mathrm{~nm})$ using the experimental setup in Figure 3a. The results in Figure 5a show that the SPR excitation angle $\theta_{e}$ localizes around $67.5^{\circ}$ for the water droplet, which corresponds to the internal angle $\theta_{i}$ of $59.6^{\circ}$. This angle is much smaller than the internal SPR angle of $\sim 70^{\circ}$ in the gold-based Kretschmann configurations, contributing to the reduction of distortion for holographic images. ${ }^{[59,67]}$ The SPR angle sensitivity can be defined as $S_{\theta}=\mathrm{d} \theta_{e} / \mathrm{d} n$. The angle sensitivity of $\mathrm{Sb}_{2} \mathrm{Te}_{3}$-based SPR is estimated as $88.5^{\circ} / \mathrm{RIU}$, which is about 1.3 (2.4) fold larger than that of traditional silver (gold)-based SPR systems. ${ }^{[57]}$ To 
achieve the real-time monitoring of the refractive index variation, we set the incident angle $\theta_{e}$ as $71.3^{\circ}\left(\theta_{i}=62^{\circ}\right)$ for the SPR excitation on the $\mathrm{Sb}_{2} \mathrm{Te}_{3}$ film with the mixture droplet. One thousand of holograms are successively recorded with a time interval of $3 \mathrm{~s}$ when the mixture volatilizes to pure water. Through the numerical reconstruction, we simultaneously obtain the intensity- and phase-contrast holographic images from the holograms for the mixture droplet. As depicted in Figure 5b, the normalized reflection increases rapidly before $600 \mathrm{~s}$ and becomes relatively flat afterwards. It is reasonable that the ethanol in the mixture quickly volatilizes in the preceding stage, and then slowly disappears. The intensity sensitivity of the $\mathrm{Sb}_{2} \mathrm{Te}_{3}$-based SPR can be defined as $S_{l}=\mathrm{d} R / \mathrm{d} n$. The intensity sensitivity strongly depends on the monitoring time and is highest around $501 \mathrm{~s}$. The average intensity sensitivity can be calculated as $\sim 15.8 \mathrm{RIU}^{-1}$. The phase differences of the reconstructed object waves correspond to the reflection phase shift differences. ${ }^{[67]}$ From Figure 5b, we can see that the reflection phase difference exhibits a nearly linear fall when the mixture droplet volatilizes from the 0 to $501 \mathrm{~s}$, and then gradually rises. In the monitoring process, the phase sensitivity is lowest at the time of $501 \mathrm{~s}$, which can be compensated by the highest intensity sensitivity. ${ }^{[59]}$ The phase difference and normalized reflection exhibit stable changes with few fluctuations, verifying the stabilization of experimental measurement. To demonstrate the process of mixture volatilization, the holograms as well as reconstructed phase and intensity images at the time of 0, 501 and 999 s are plotted in Figure 5c-e. The holograms possess typical interference patterns, as depicted in the inset of Figure 5c. The intensity of hologram in the area of mixture droplet is relatively dark owing to the SPR excitation at first and then distinctly increases with the monitoring time. Moreover, the phase experiences a descending process 
from $0 \mathrm{~s}$ to $501 \mathrm{~s}$, and then increases from $501 \mathrm{~s}$ to $999 \mathrm{~s}$ (Figure 5d). As shown in Figure 5e, the reconstructed intensity of the mixture droplet first rises and then descends, which is in accordance with the holograms. Additionally, all the images show that the droplet shrinks because the ethanol volatilizes. These results agree well with the alternations of reflection and phase in Figure 5b. Also, it should be noted that the images of droplets are less distorted because of the relatively small incident angle. The measuring value of refractive index in the $\mathrm{Sb}_{2} \mathrm{Te}_{3}$-based SPR system can approach 1.454 when the incident wavelength is $640 \mathrm{~nm}$ (Supporting Information). The measurement range is improved by $24.4 \%$, compared to those of the gold- and silver-based SPR systems. The improvement of measurement range will increase when the incident wavelength decreases and exceeds $50 \%$ at $400 \mathrm{~nm}$ wavelength.

In summary, we have experimentally presented the generation of the SPR on the topological insulator $\mathrm{Sb}_{2} \mathrm{Te}_{3}$ film at visible frequencies. The results demonstrate that the $\mathrm{Sb}_{2} \mathrm{Te}_{3}$-based SPR can be effectively excited with the incident angle smaller than the traditional metal-based SPR. The experiment results are in good agreement with the theoretical calculations. Based on $\mathrm{Sb}_{2} \mathrm{Te}_{3}$-based SPR, we develop an impactful digital holographic system to significantly realize the real-time, full-view, distortion-reduced and nondestructive monitoring of near-field refractive index variation. It is found that the $\mathrm{Sb}_{2} \mathrm{Te}_{3}$-based SPR has the particular advantage of broader measurement range, compared with the traditional metal-based SPR. Our results not only reveal the plasmonic characteristics of the $\mathrm{Sb}_{2} \mathrm{Te}_{3}$ topological insulator at high frequencies, but also pave a new avenue for significant applications of topological insulators in photonics and optoelectronics, especially for environmental and biochemical sensing. 


\section{Experimental Section}

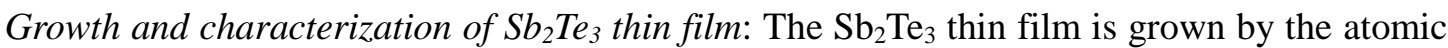
layer deposition (ALD) system, which enables to fabricate large-area optical films with nanometer precision at the relatively low temperature. $\mathrm{Sb}\left(\mathrm{OC}_{2} \mathrm{H}_{5}\right)_{3}+3\left[\left(\mathrm{CH}_{3}\right)_{3} \mathrm{Si}\right]_{2} \mathrm{Te} \rightarrow \mathrm{Sb}_{2} \mathrm{Te}_{3}+$ $6\left(\mathrm{CH}_{3}\right)_{3} \mathrm{Si}-\mathrm{OC}_{2} \mathrm{H}_{5}$ is the chemical equation employed to synthesize the $\mathrm{Sb}_{2} \mathrm{Te}_{3}$ material. ${ }^{[52]}$ For the precursor of the chemical material $\mathrm{Sb}\left(\mathrm{OC}_{2} \mathrm{H}_{5}\right)_{3}$, the injection (purge) time is set as 0.75 (9) s. For the precursor of $3\left[\left(\mathrm{CH}_{3}\right)_{3} \mathrm{Si}\right]_{2} \mathrm{Te}$, the injection (purge) time is set as 0.1 (9) s. The precursor transport and substrate temperatures are controlled at 150 and $70{ }^{\circ} \mathrm{C}$, respectively. The ALD growth time is set as 5 hours for the deposition of a $50 \mathrm{~nm} \mathrm{Sb} \mathrm{Te}_{3}$ film. The SEM (FEI Quanta FEG 250) image is used to reveal the surface profile of the grown topological insulator film on the glass. The thickness of $\mathrm{Sb}_{2} \mathrm{Te}_{3}$ film can be verified by the $\mathrm{AFM}$ (Bruker Dimension FastScan) image. The $\mathrm{Sb}_{2} \mathrm{Te}_{3}$ stoichiometry of the film can be confirmed by Raman shift spectrum. The SPP-enhanced Raman spectrum cannot be generated when the laser beam directly impinges on the $\mathrm{Sb}_{2} \mathrm{Te}_{3}$ film. The crystallinity of $\mathrm{Sb}_{2} \mathrm{Te}_{3}$ film is confirmed by XRD (Shimadzu XRD-7000).

Calculations of SPR angles: The glass substrate, matching liquid, prism can be treated as one layer due to their very close refractive indices. Thus, the Kretschmann configuration for the $\mathrm{SPR}$ generation can be regarded as a five-layer (air, $\mathrm{Sb}_{2} \mathrm{Te}_{3}$ surface layer, $\mathrm{Sb}_{2} \mathrm{Te}_{3}$ bulk layer, $\mathrm{Sb}_{2} \mathrm{Te}_{3}$ surface layer, and glass substrate) photonic structure. According to Maxwell's equations and boundary conditions of different optical layers, ${ }^{[68]}$ the SPR excitation angle $\theta_{i}$ can be calculated by solving the equations 


$$
\begin{gathered}
0=\left(1+Q_{04}\right)+\left(Q_{01}+Q_{14}\right) P_{1}+\left(Q_{02}+Q_{24}\right) P_{2}+\left(Q_{03}+Q_{34}\right) P_{3} \\
+\left(Q_{12}+Q_{01} Q_{24}\right) P_{1} P_{2}+\left(Q_{13}+Q_{01} Q_{34}\right) P_{1} P_{3}+\left(Q_{23}+Q_{02} Q_{34}\right) P_{2} P_{3} \\
+\left(Q_{01} Q_{23}+Q_{12} Q_{34}\right) P_{1} P_{2} P_{3} \\
Q_{m n}=\frac{\varepsilon_{m} k_{0}\left(n_{e f f}^{2}-\varepsilon_{n}\right)}{\varepsilon_{n} k_{0}\left(n_{e f f}^{2}-\varepsilon_{m}\right)} \quad(m, n=0,1,2,3,4) \\
P_{m}=\tanh \left[d_{m} k_{0}\left(n_{e f f}^{2}-\varepsilon_{m}\right)\right] \quad(m=1,2,3) \\
\theta_{i}=\arcsin \left(\frac{n_{e f f}}{n_{4}}\right),
\end{gathered}
$$

where $\varepsilon_{0}(=1), \varepsilon_{1}\left(=\varepsilon_{3}=\varepsilon_{s}\right), \varepsilon_{2}\left(=\varepsilon_{b}\right)$ and $\varepsilon_{4}\left(=n^{2} \approx 2.3\right)$ are the dielectric constants of the air, $\mathrm{Sb}_{2} \mathrm{Te}_{3}$ surface layer, $\mathrm{Sb}_{2} \mathrm{Te}_{3}$ bulk layer, and substrate, respectively. $k_{0}=2 \pi / \lambda$ is the wave number of incident light in vacuum. The thickness of topological surface state is considered as about $1.5 \mathrm{~nm} .{ }^{[45,52]}$ Thus, $d_{1}\left(=d_{3}=1.5 \mathrm{~nm}\right)$ and $d_{2}(=47 \mathrm{~nm})$ stand for the thicknesses of the surface and bulk states in the $\mathrm{Sb}_{2} \mathrm{Te}_{3}$ film, respectively. The external excitation angle $\theta_{e}$ can be obtained as

$$
\theta_{e}=\left\{\begin{array}{ll}
45^{o}-\arcsin \left[n_{4} \sin \left(45^{\circ}-\theta_{i}\right)\right] & \left(\theta_{i}<45^{\circ}\right) \\
45^{o}+\arcsin \left[n_{4} \sin \left(\theta_{i}-45^{\circ}\right)\right] & \left(\theta_{i} \geq 45^{\circ}\right)
\end{array} .\right.
$$

FDTD simulations: The FDTD method is utilized to numerically simulate the SPR field distribution on the $\mathrm{Sb}_{2} \mathrm{Te}_{3}$ thin film. In the simulations, the perfectly matched layer absorbing boundary condition is set at the top/bottom of computational space. ${ }^{[69]}$ The Bloch boundary condition is set at the right/left sides for the oblique incidence of light. To make the grids of surface layers in the $\mathrm{Sb}_{2} \mathrm{Te}_{3}$ film sufficiently fine, the non-uniform mesh is applied in the direction perpendicular to the $\mathrm{Sb}_{2} \mathrm{Te}_{3}$ film. The maximum mesh size is set as $0.2 \mathrm{~nm}$ in the surface layers. In the direction parallel to the $\mathrm{Sb}_{2} \mathrm{Te}_{3}$ film, the mesh size is uniformly set as 5 nm. The dielectric constants of surface and bulk layers in the $\mathrm{Sb}_{2} \mathrm{Te}_{3}$ film are separately imported into the simulation models. 


\section{Supporting Information}

Supporting Information is available from the Wiley Online Library or from the author.

\section{Acknowledgements}

This work was supported by the National Key R\&D Program of China (2017YFA0303800), National Natural Science Foundation of China (11634010, 61705186, and 11774290), Natural Science Basic Research Plan in Shaanxi Province of China (2017JQ1023), Technology Foundation for Selected Overseas Chinese Scholar of Shaanxi Province (2017007) and Fundamental Research Funds for the Central Universities (3102018zy039 and 3102018zy050). The materials were fabricated at Melbourne Centre for Nanofabrication. The authors thanks Prof. Min Gu from RMIT University for the support in the material fabrication. The authors also thank Dr Jiwei Zhang, Chenyang Zhao, Teli Xi, and Ruijuan Tian for the technical assistances in the digital holography, AFM measurement, 3D structure, and Raman spectrum measurement, respectively. The authors thank Changchun New Industries Optoelectronics Technology Co., Ltd. for the support of semiconductor lasers. The authors also thank the Analytical \& Testing Center of Northwestern Polytechnical University for the AFM, SEM, $\mathrm{XRD}$, and EDS measurement.

\section{Conflict of Interest}

The authors declare no conflict of interest.

\section{Keywords}

topological insulators, surface plasmon resonance, digital holography, refractive index monitoring 


\section{References}

[1] W. Barnes, A. Dereux, T. Ebbesen, Nature 2003, 424, 824.

[2] S. Maier, Plasmonics: Fundamentals and Applications. Springer, 2007.

[3] D. Gramotnev, S. Bozhevolnyi, Nat. Photonics 2010, 4, 83.

[4] A. Naldoni, V. Shalaev, M. Brongersma, Science 2017, 356, 908.

[5] N. Jiang, X. Zhuo, J. Wang, Chem. Rev. 2018, 118, 3054.

[6] D. Lei, K. Appavoo, F. Ligmajer, Y. Sonnefraud, R. Jr, S. Maier, ACS Photonics 2015, 2, 1306.

[7] X. Li, L. Chen, Y. Li, X. Zhang, M. Pu, Z. Zhao, X. Ma, Y. Wang, M. Hong, X. Luo, Sci. Adv. 2016, 2, e1601102.

[8] Z. Li, Adv. Opt. Mater. 2018, 1701097.

[9] P. Yu, J. Li, C. Tang, H. Cheng, Z. Liu, Z. Li, Z. Liu, C. Gu, J. Li, S. Chen, J. Tian, Light Sci. Appl. 2016, 5, e16096.

[10] S. Liu, E. Leong, G. Li, Y. Hou, J. Deng, J. Teng, H. Ong, D. Lei, ACS Nano 2016, 10, 1442.

[11] C. Min, Z. Shen, J. Shen, Y. Zhang, H. Fang, G. Yuan, L. Du, S. Zhu, T. Lei, X. Yuan, Nat. Commun. 2013, 4, 2891.

[12] S. Wang, X. Wang, B. Li, H. Chen, Y. Wang, L. Dai, R. Oulton, R. Ma, Nat. Commun. 2017, 8, 1889.

[13] Z. Deng, J. Deng, X. Zhuang, S. Wang, K. Li, Y. Wang, Y. Chi, X. Ye, J. Xu, G. Wang, R. Zhao, X. Wang, Y. Cao, X. Cheng, G. Li, X. Li, Nano Lett. 2018, 18, 2885.

[14] S. Wang, Q. Van, T. Peyronel, M. Ramezani, N. Hoof, T. Tiecke, J. Rivas, ACS Photonics 
2018, 5, 2478.

[15] H. Liu, P. Lalanne, Nature 2008, 452, 728.

[16] P. Lalanne, W. Yan, K. Vynck, C. Sauvan, J. Hugonin, Laser Photon. Rev. 2018, 12, 1700113.

[17] J. Ou, E. Plum, J. Zhang, N. Zheludev, Adv. Mater. 2016, 28, 729.

[18] S. Chen, Z. Li, Y. Zhang, H. Cheng, J. Tian, Adv. Opt. Mater. 2018, 6, 1800104.

[19] N. Liu, L. Langguth, T. Weiss, J. Kästel, M. Fleischhauer, T. Pfau, H. Giessen, Nat. Mater. 2009, 8, 758 .

[20] R. Taubert, M. Hentschel, J. Kastel, H. Giessen, Nano Lett. 2012, 12, 1367.

[21] X. Liu, J. Kang, H. Yuan, J. Park, S. J. Kim, Y. Cui, H. Hwang, M. Brongersma, Nat. Nanotechnol. 2017, 12, 866.

[22] J. F. Li, J. Mu, B. Wang, W. Ding, J. Liu, H. Guo, W. Li, C. Gu, Z. Li, Laser Photon. Rev. 2014, 8, 602 .

[23] M. Papaioannou, E. Plum, E. Rogers, N. Zheludev, Light Sci. Appl. 2018, 7, 17157.

[24] Y. J. Li, Y. Lv, C. Zou, W. Zhang, J. Yao, Y. Zhao, J. Am. Chem. Soc. 2016, 138, 2122.

[25] J. Li, P. Yu, C. Tang, H. Cheng, J. Li, S. Chen, J. Tian, Adv. Opt. Mater. 2017, 5, 1700152.

[26] Z. Chai, Y. Zhu, X. Hu, X. Yang, Z. Gong, F. Wang, H. Yang, Q. Gong, Adv. Opt. Mater. 2016, 4, 1159.

[27] L. Shao, X. Zhuo, J. Wang, Adv. Mater. 2018, 30, 1704338.

[28] Y. Yan, C. Zhang, J. Y. Zheng, J. Yao, Y. S. Zhao, Adv. Mater. 2012, 24, 5681.

[29] S. Chen, Y. Zhang, T. Shih, W. Yang, S. Hu, X. Hu, J. Li, B. Ren, B. Mao, Z. Yang, Z. 
Tian. Nano Lett. 2018, 18, 2209.

[30] H. Lu, X. Liu, D. Mao, G. Wang, Opt. Lett. 2012, 37, 3780.

[31] G. Naik, V. Shalaev, A. Boltasseva, Adv. Mater. 2013, 25, 3264.

[32] A. Grigorenko, M. Polini, K. Novoselov, Nat. Photonics. 2012, 6, 749.

[33] Q. Xu, T. Ma, M. Danesh, B. Shivananju, S. Gan, J. Song, C. Qiu, H. Cheng, W. Ren, Q. Bao, Light Sci. Appl. 2017, 6, e16204.

[34] W. Luo, W. Cai, W. Wu, Y. Xiang, M. Ren, X. Zhang, J. Xu, 2D Mater. 2016, 3, 045001.

[35] H. Lu, C. Zeng, Q. Zhang, X. Liu, M. Hossain, P. Reineck M. Gu, Sci. Rep. 2015, 5, 8443.

[36] H. Yan, T. Low, W. Zhu, Y. Wu, M. Freitag, X. Li, F. Guinea, P. Avouris, F. Xia, Nat. Photonics 2013, 7, 394.

[37] R. Yu, R. Alaee, F. Lederer, C. Rockstuhl, Phys. Rev. B 2014, 90, 085409.

[38] Y. Wang, J. Ou, A. Chrimes, B. Carey, T. Daeneke, M. Alsaif, M. Mortazavi, S. Zhuiykov, N. Medhekar, M. Bhaskaran, J. Friend, M. Strano, K. Zadeh, Nano Lett. 2015, 15, 883.

[39] Z. Liu, K. Aydin, Nano Lett. 2016, 16, 3457.

[40] T. Low, R. Roldan, H. Wang, F. Xia, P. Avouris, L. Moreno, F. Guinea, Phys. Rev. Lett. 2014, 113, 106802.

[41] P. Pietro, M. Ortolani, O. Limaj, A. Gaspare, V. Giliberti, F. Giorgianni, M. Brahlek, N. Bansal, N. Koirala, S. Oh, P. Calvani, S. Lupi, Nat. Nanotechnol. 2013, 8, 556.

[42] M. Autore, H. Engelkamp, F. Apuzzo, A. Gaspare, P. Pietro, I. Vecchio, M. Brahlek, N. Koirala, S. Oh, S. Lupi, ASC Photonics 2015, 2, 1231.

[43] M. Zhao, M. Bosman, M. Danesh, M. Zeng, P. Song, Y. Darma, A. Rusydi, H. Lin, C. 
Qiu, K. Loh, Nano Lett. 2015, 15, 8331.

[44] J. Yuan, W. Ma, L. Zhang, Y. Lu, M. Zhao, H. Guo, J. Zhao, W. Yu, Y. Zhang, K. Zhang, H. Hoh, X. Li, K. Loh, S. Li, C. Qiu, Q. Bao, ACS Photonics 2017, 4, 3055.

[45] J. Ou, J. So, G. Adamo, A. Sulaev, L. Wang, N. Zheludev, Nat. Commun. 2014, 5, 5139.

[46] A. Dubrovkin, G. Adamo, J. Yin, L. Wang, C. Soci, Q. Wang, N. Zheludev, Adv. Opt. Mater. 2017, 5, 1600768.

[47] J. Yin, H. Krishnamoorthy, G. Adamo, A. Dubrovkin, Y. Chong, N. Zheludev, C. Soci, NPG Asia Mater. 2017, 9, e425.

[48] Z. Yue, B. Cai, L. Wang, X. Wang, M. Gu, Sci. Adv. 2016, 2, e1501536.

[49] S. Sim, H. Jang, N. Koirala, M. Brahlek, J. Moon, J. Sung, J. Park, S. Cha, S. Oh, M. Jo, J. Ahn, H. Choi, Nat. Commun. 2015, 6, 9814.

[50] M. Zhao, J. Zhang, N. Gao, P. Song, M. Bosman, B. Peng, B. Sun, C. Qiu, Q. Xu, Q. Bao, K. Loh, Adv. Mater. 2016, 28, 3138.

[51] H. Zhang, C. Liu, X. Qi, X. Dai, Z. Fang, S. Zhang, Nat. Phys. 2009, 5, 438.

[52] Z. Yue, G. Xue, J. Liu, Y. Wang, M. Gu, Nat. Commun. 2017, 8, 15354.

[53] M. Kowalczyk, J. Bogusławski, R. Zybała, K. Mars, A. Mikuła, G. Soboń, J. Sotor, Opt. Mater. Express 2016, 6, 2273.

[54] E. Goi, Z. Yue, B. Cumming, M. Gu, Laser Photon. Rev. 2018, 12, 1700271.

[55] A. Zayats, I. Smolyaninov, J. Opt. A: Pure Appl. Opt. 2003, 5, S16.

[56] H. Jussila, H. Yang, N. Granqvist, Z. Sun, Optica 2016, 3, 151.

[57] A. Abbas, M. Linman, Q. Cheng, Sens. Actuators B: Chem. 2011, 156, 169.

[58] A. Kabashin, S. Patskovsky, A. Grigorenko, Opt. Express 2009, 17, 21191. 
[59] S. Li, J. Zhong, Biomed. Opt. Express 2012, 3, 3190.

[60] J. Homola, Chem. Rev. 2008, 108, 462.

[61] G. Jia, W. Lou, F. Cheng, X. Wang, J. Yao, N. Dai, H. Lin, K. Chang, Nano Res. 2015, 8, 1443.

[62] G. Jia, P. Wang, Y. Zhang, K. Chang, Sci. Rep. 2016, 6, 25884.

[63] Z. Sekkat, S. Hayashi, D. V. Nesterenko, A. Rahmouni, S. Refki, H. Ishitobi, Y. Inouye, S. Kawata, Opt. Express 2016, 24, 20080.

[64] K. Matsunaga, Y. Hirai, Y. Neo, T. Matsumoto, M. Tomita, Sci. Rep. 2017, 7, 17824.

[65] Z. Wu, F. Peeters, K. Chang, Phys. Rev. B 2010, 82, 115211.

[66] J. Zhu, D. Yao, S. Zhang, K. Chang, Phys. Rev. Lett. 2011, 106, 097201.

[67] J. Zhang, C. Ma, S. Dai, J. Di, Y. Li, T. Xi, J. Zhao, Opt. Lett. 2016, 41, 3844.

[68] X. Kong, W. Yan, Z. Li, J. Tian, Opt. Express 2012, 20, 12133.

[69] A. Taflove, S. Hagness, Computational Electrodynamics: The Finite-Difference Time-Domain Method. 2nd ed. Artech House, Boston 2000. 


\section{Figure legends}

(a)
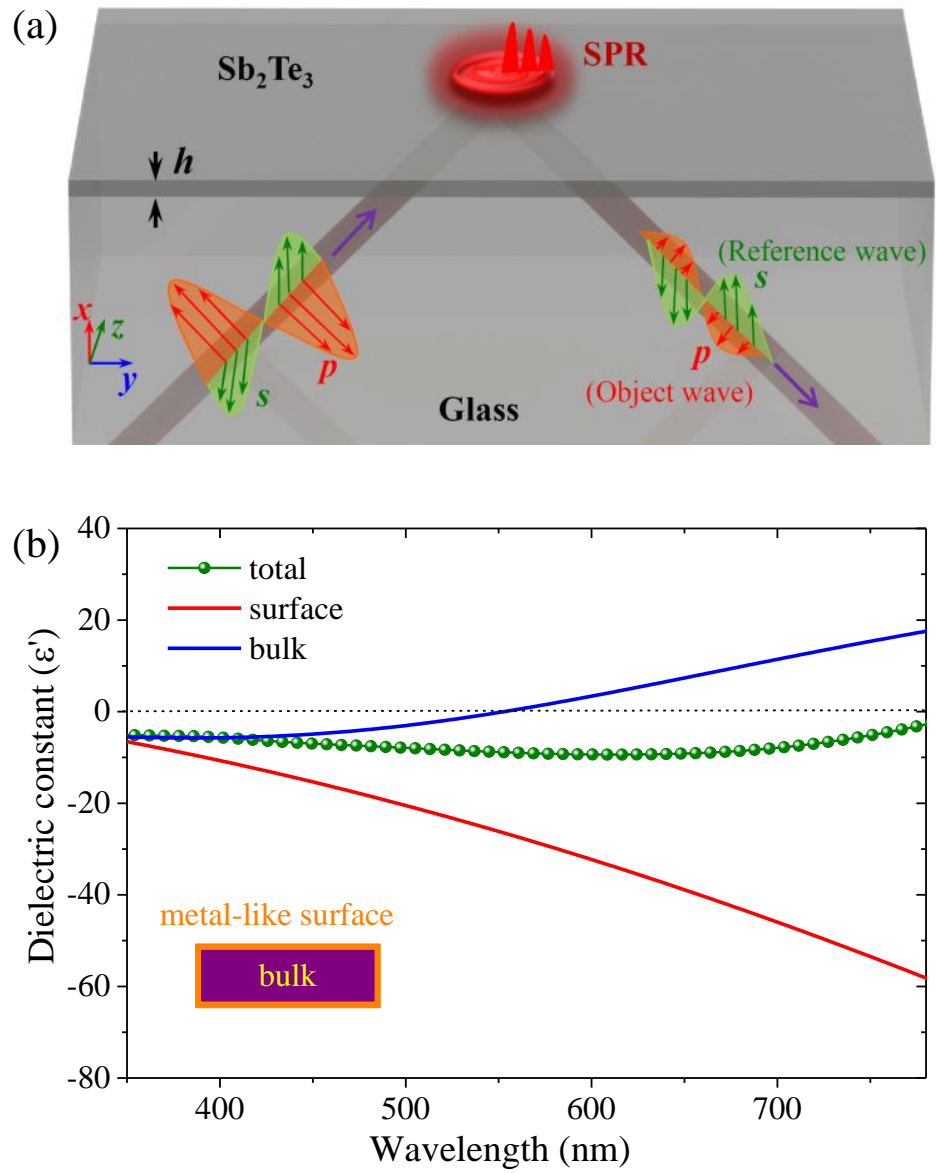

Figure 1. Deposited $\mathrm{Sb}_{2} \mathrm{Te}_{3}$ topological insulator film and dielectric constants of $\mathrm{Sb}_{2} \mathrm{Te}_{3}$ material. (a) Schematic illustration of the $\mathrm{Sb}_{2} \mathrm{Te}_{3}$ topological insulator thin film grown by ALD deposition. The $\mathrm{Sb}_{2} \mathrm{Te}_{3}$ film is grown on the glass with a thickness of $50 \mathrm{~nm}(h=50 \mathrm{~nm})$.

(b) Real parts of wavelength-dependent dielectric constants of the $\mathrm{Sb}_{2} \mathrm{Te}_{3}$ material in the visible region, which are measured using the spectroscopic ellipsometer (J. A. Woollam Co.). The inset shows the surface-on-bulk model of $\mathrm{Sb}_{2} \mathrm{Te}_{3}$ topological insulator. 

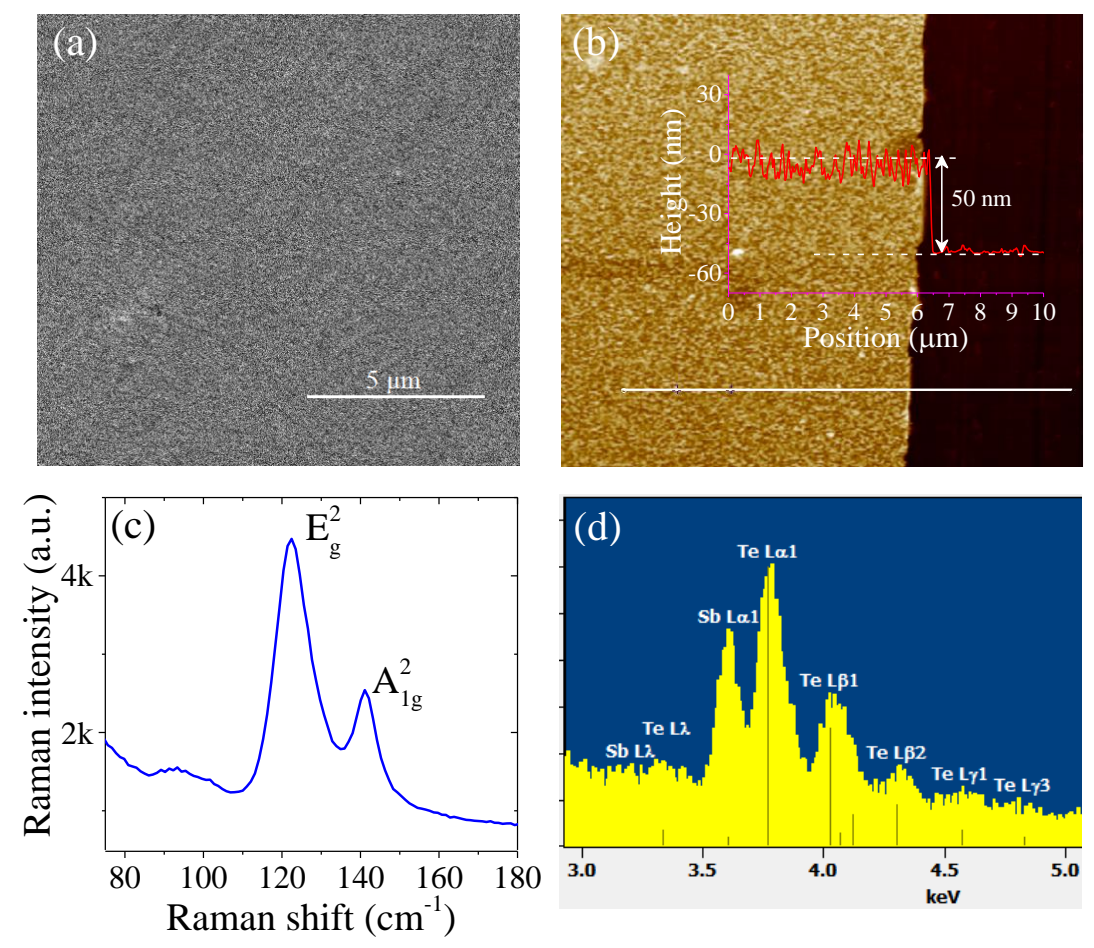

Figure 2. (a) Top-view scanning electron microscope (SEM) image of the $\mathrm{Sb}_{2} \mathrm{Te}_{3}$ film. (b) Atomic force microscope (AFM) image of the $\mathrm{Sb}_{2} \mathrm{Te}_{3}$ film with a part of naked glass. The white solid line denotes the position for AFM height measurement. The inset shows the heights of the film along the white line. (c) Raman spectroscopy of the $\mathrm{Sb}_{2} \mathrm{Te}_{3}$ film with impinging a laser beam with a wavelength of $532 \mathrm{~nm}$. (d) Energy-dispersive X-ray spectroscopy (EDS) of the $\mathrm{Sb}_{2} \mathrm{Te}_{3}$ film. 
(a)
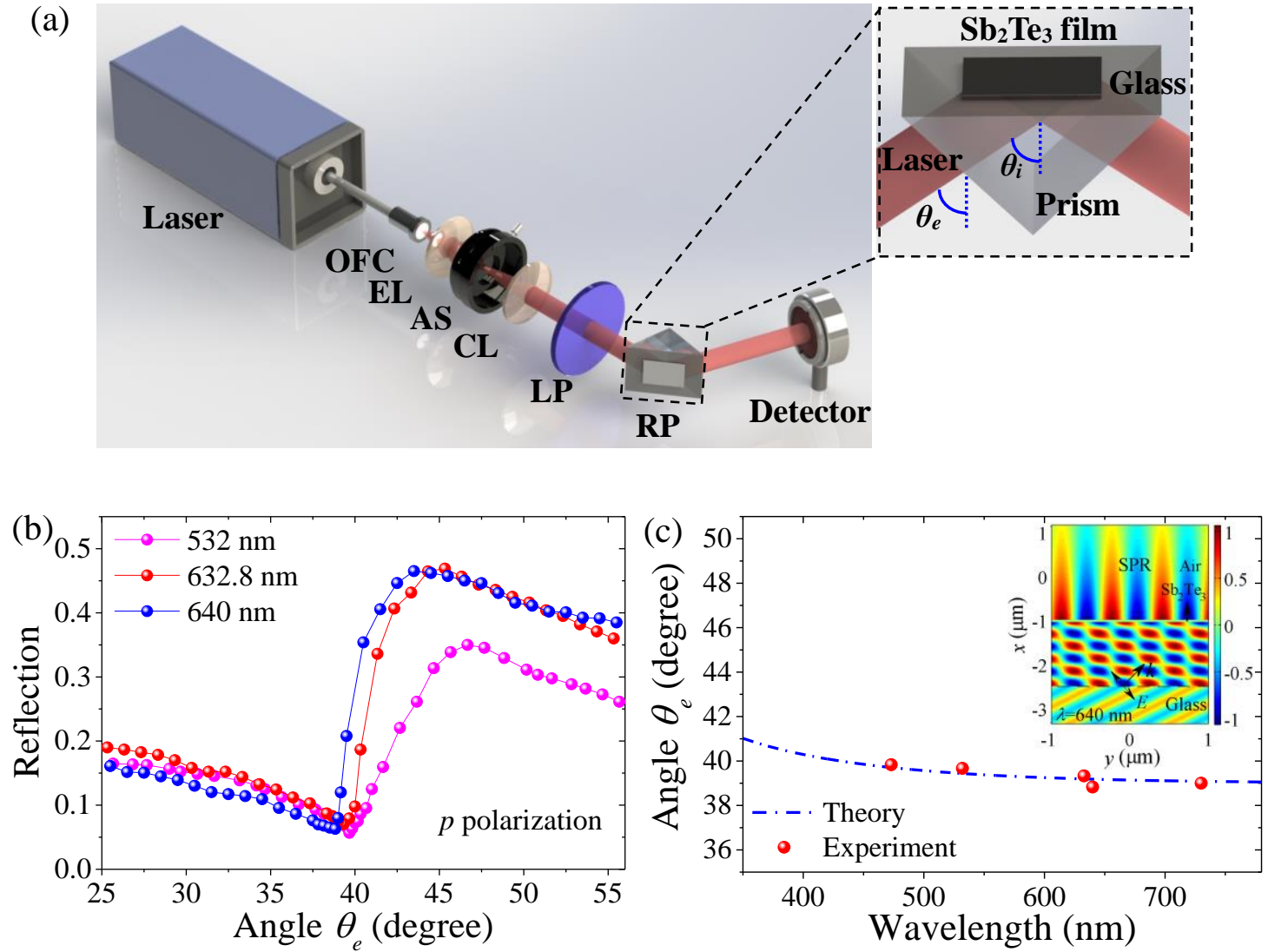

Figure 3. (a) Experimental setup for the generation of $\mathrm{SPR}$ on the $\mathrm{Sb}_{2} \mathrm{Te}_{3}$ thin film. The inset depicts the rectangular prism with a $\mathrm{Sb}_{2} \mathrm{Te}_{3}$ film on glass slip. The external and internal incident angles of laser beam on the prism are $\theta_{e}$ and $\theta_{i}$, respectively. Here, OFC: optical fiber coupler, EL: expanding lens, AS: aperture stop, CL: collimator lens, LP: linear polarizer and RP: rectangular prism. (b) Experiment results of light reflection in the $\mathrm{Sb}_{2} \mathrm{Te}_{3}$-based Kretschmann configuration with different incident angles $\theta_{e}$ at the wavelengths of $532,632.8$, and $640 \mathrm{~nm}$ for the p-polarized light. (c) Excitation angles of the SPR on the $\mathrm{Sb}_{2} \mathrm{Te}_{3}$ thin film at different wavelengths. The curve and circles denote the theoretical and experimental data, respectively. The inset shows the simulation result of $E_{y}$ field distribution in the $\mathrm{Sb}_{2} \mathrm{Te}_{3}$ film structure with obliquely illuminating a $640 \mathrm{~nm}$ plane wave at the angle of the SPR excitation. 

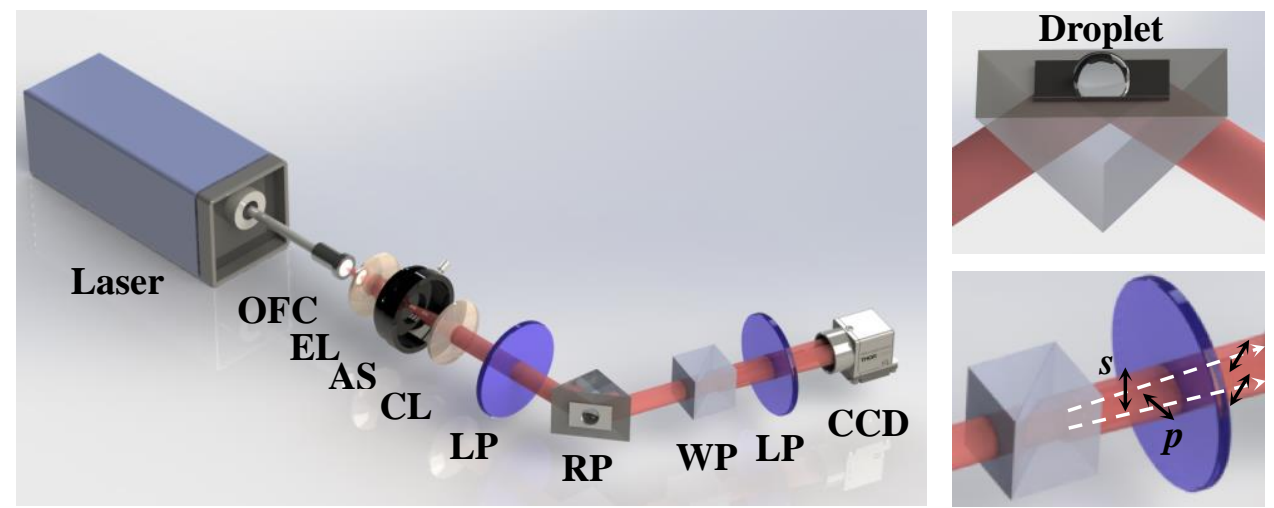

Figure 4. Experimental setup of the developed common-path DH combining with the SPR on the $\mathrm{Sb}_{2} \mathrm{Te}_{3}$ thin film for the measurement of refractive index variation. Here, WP: Wollaston prism and CCD: charge-coupled device. The other symbols are identical with those depicted in Figure 2(a). The upper-right inset shows the prism with the $\mathrm{Sb}_{2} \mathrm{Te}_{3}$ thin film adhered by a mixture droplet. The lower-right inset depicts the light beam splitting of $s$ - and $p$-polarized components through the WP. 

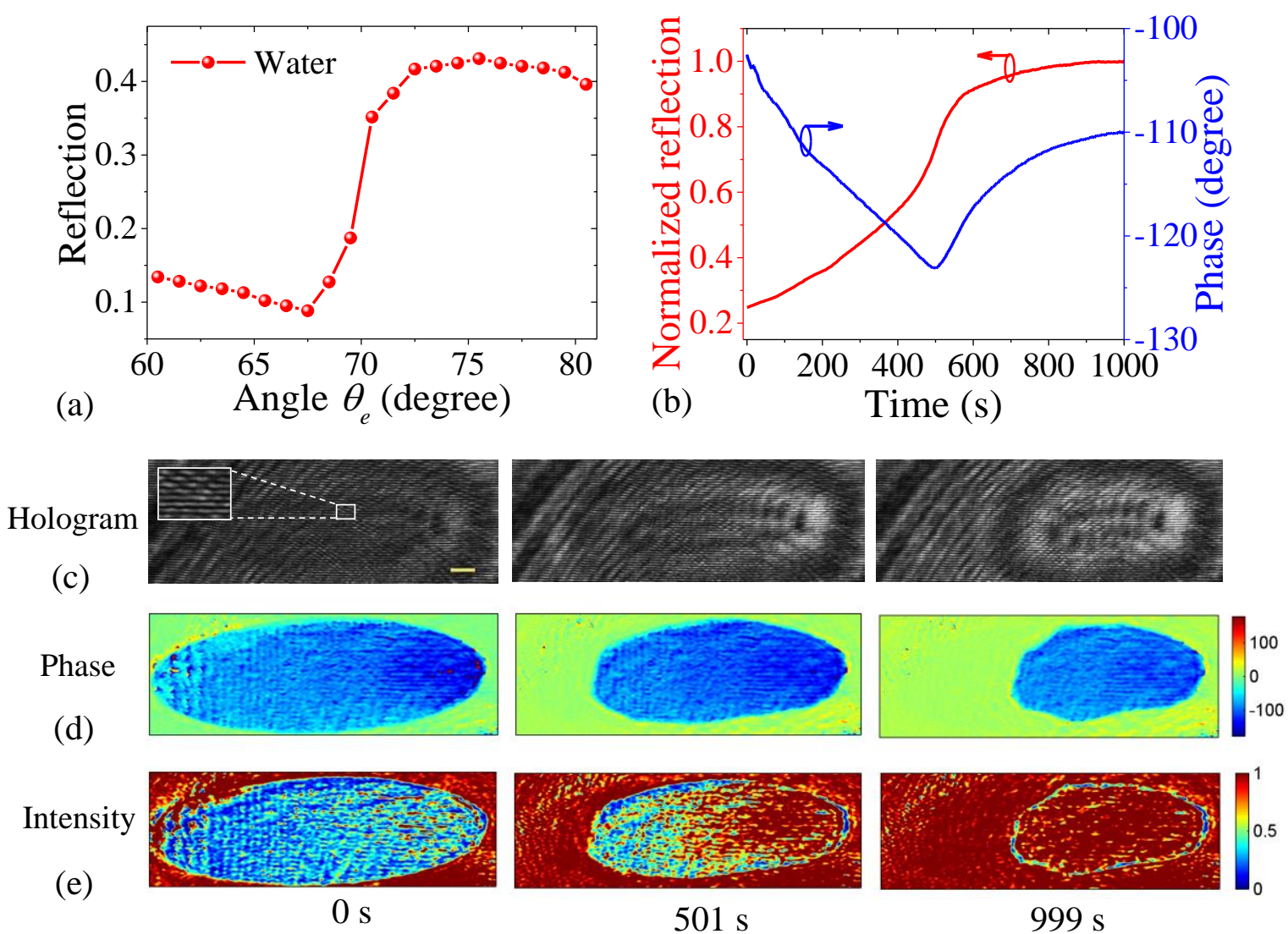

Figure 5. (a) Reflection of the light beam at different incident angles in the $\mathrm{Sb}_{2} \mathrm{Te}_{3}$-based

Kretschmann configuration with a deionized water droplet. (b) Normalized intensity reflection and phase shift difference of the reconstructed images at different time. (c)-(e) Holograms, reconstructed phases and reconstructed intensities when $t=0 \mathrm{~s}, 501 \mathrm{~s}$, and $999 \mathrm{~s}$. The scale bar is $200 \mu \mathrm{m}$ in (c). The inset in (c) depicts the zoom-in view of interference pattern for the hologram in the area of mixture droplet. 